\title{
Star versions of Hurewicz spaces
}

\author{
Sumit Singh $^{1}$ (D), Ljubiša D.R. Kočinac ${ }^{* 2}$ (D) \\ ${ }^{1}$ Department of Mathematics, University of Delhi, New Delhi-110007, India \\ ${ }^{2}$ University of Niš, Faculty of Sciences and Mathematics, 18000 Niš, Serbia
}

\begin{abstract}
A space $X$ is said to have the set star Hurewicz property if for each nonempty subset $A$ of $X$ and each sequence $\left(\mathcal{U}_{n}: n \in \mathbb{N}\right)$ of collections of sets open in $X$ such that for each $n \in \mathbb{N}, \bar{A} \subset \cup \mathcal{U}_{n}$, there is a sequence $\left(\mathcal{V}_{n}: n \in \mathbb{N}\right)$ such that for each $n \in \mathbb{N}, \mathcal{V}_{n}$ is a finite subset of $\mathcal{U}_{n}$ and for each $x \in A, x \in \operatorname{St}\left(\cup \mathcal{V}_{n}, \mathcal{U}_{n}\right)$ for all but finitely many $n$. In this paper, we investigate the relationships among set star Hurewicz, set strongly star Hurewicz and other related covering properties and study the topological properties of these topological spaces.
\end{abstract}

Mathematics Subject Classification (2020). 54D20, 54B05, 54B10, 54C10, 54D99

Keywords. Hurewicz, star Hurewicz, strongly star Hurewicz, set star Hurewicz, set-SSH

\section{Introduction and preliminaries}

In [1], Arhangel'skii defined a cardinal function $s L$, and spaces $X$ such that $s L(X)=\omega$ we call s-Lindelöf: a space $X$ is s-Lindelöf if for each subset $A$ of $X$ and each cover $\mathcal{U}$ of $\bar{A}$ by sets open in $X$ there is a countable set $\mathcal{V} \subset \mathcal{U}$ such that $A \subset \overline{U \mathcal{V}}$. Motivated by this definition, and modifying it, Kočinac and Konca [12] considered new types of selective covering properties called set covering properties. Later on, Kočinac, Konca and Singh in [13] studied set star covering properties using the star operator, and, in particular, defined set star Hurewicz and set strongly star Hurewicz properties.

In this paper, we investigate the relationship among set star Hurewicz, set strongly star Hurewicz and other related properties. Further, we study the topological properties of these two classes of spaces.

Throughout the paper we use standard topological terminology and notation as in [6]. By "a space" we mean "a topological space", $\mathbb{N}$ denotes the set of natural numbers, and an open cover $\mathcal{U}$ of a subset $A \subset X$ means elements of $\mathcal{U}$ are open in $X$ and $A \subset \cup \mathcal{U}=$ $\cup\{U: U \in \mathcal{U}\}$. The cardinality of a set $A$ is denoted by $|A|$. Let $\omega$ denote the first infinite cardinal, $\omega_{1}$ the first uncountable cardinal, $\mathfrak{c}$ the cardinality of the set of real numbers. As usual, a cardinal is an initial ordinal and an ordinal is the set of smaller ordinals. A cardinal is often viewed as a space with the usual order topology. If $A$ is a subset of a space $X$ and $\mathcal{U}$ is a collection of subsets of $X$, then the star of $A$ with respect to $\mathcal{U}$ is the

\footnotetext{
*Corresponding Author.

Email addresses: sumitkumar405@gmail.com (S. Singh), lkocinac@gmail.com (Lj.D.R. Kočinac)

Received: 02.11.2020; Accepted: 08.04.2021
} 
set $\operatorname{St}(A, \mathcal{U}):=\bigcup\{U \in \mathcal{U}: U \cap A \neq \emptyset\}$; also, if $x \in X$, then we just write $\operatorname{St}(x, \mathcal{U})$ instead of $\operatorname{St}(\{x\}, \mathcal{U})$.

We first recall the classical notions of spaces which are used in this paper.

In 1925, Hurewicz $[7,8]$ introduced the Hurewicz covering property for a space $X$ in the following way:

A space $X$ is said to have the Hurewicz property if each sequence $\left(\mathcal{U}_{n}: n \in \mathbb{N}\right)$ of open covers of $X$ there is a sequence $\left(\mathcal{V}_{n}: n \in \mathbb{N}\right)$ such that for each $n \in \mathbb{N}, \mathcal{V}_{n}$ is a finite subset of $\mathcal{U}_{n}$ and for each $x \in X, x \in \cup \mathcal{V}_{n}$ for all but finitely many $n$.

Kočinac $[3,9,10]$, introduced the star versions of the Hurewicz covering property using the star operator in the following way:

(1) A space $X$ is said to have the star Hurewicz property (shortly, SH property) if each sequence $\left(\mathcal{U}_{n}: n \in \mathbb{N}\right)$ of open covers of $X$, there is a sequence $\left(\mathcal{V}_{n}: n \in \mathbb{N}\right)$ such that for each $n \in \mathbb{N}, \mathcal{V}_{n}$ is a finite subset of $\mathcal{U}_{n}$ and for each $x \in X, x \in \operatorname{St}\left(\cup \mathcal{V}_{n}, \mathcal{U}_{n}\right)$ for all but finitely many $n$.

(2) A space $X$ is said to have the strongly star Hurewicz property (shortly, SSH property) if for each sequence $\left(\mathcal{U}_{n}: n \in \mathbb{N}\right)$ of open covers of $X$, there is a sequence $\left(F_{n}: n \in \mathbb{N}\right)$ of finite subsets of $X$ such that for each $x \in X, x \in \operatorname{St}\left(F_{n}, \mathcal{U}_{n}\right)$ for all but finitely many $n$.

In what follows we will use Theorem 1.1 below.

Recall that a collection $\mathcal{A}$ of infinite subsets of $\omega$ is said to be almost disjoint if the sets $A \cap B$ are finite for all distinct elements $A, B \in \mathcal{A}$. For an almost disjoint family $\mathcal{A}$, put $\psi(\mathcal{A})=\mathcal{A} \cup \omega$ and topologize $\psi(\mathcal{A})$ as follows: all points in $\omega$ are isolated, and for each $A \in \mathcal{A}$ and each finite set $F \subset \omega,\{A\} \cup(A \backslash F)$ is a basic open neighborhood of $A$. The spaces of this type are called Isbell-Mrówka $\psi$-spaces $[2,6,15]$ or $\psi(\mathcal{A})$ spaces.

Theorem $1.1([2])$. Let $\mathcal{A}$ be an almost disjoint family of infinite subsets of $\omega$ and let $\psi(\mathcal{A})=\omega \cup \mathcal{A}$ be the Isbell-Mrówka space. Then:

(1) $\psi(\mathcal{A})$ is strongly star Hurewicz if and only if $|\mathcal{A}|<\mathfrak{b}$;

(2) If $|\mathcal{A}|=\mathfrak{c}$, then $\psi(X)$ is not star Hurewicz.

Recently, Kočinac and Konca [12] defined the set selection properties (and their weak versions). See also the paper $[11,16]$ related to these properties.

In [13], Kočinac, Konca and Singh defined (general versions of) set star selection properties and, in particular, the set star Hurewicz and set strongly star Hurewicz spaces.

Definition 1.2. A space $X$ is said to have the

(1) set star Hurewicz property (shortly, set-SH property) if for each nonempty set $A$ of $X$ and each sequence $\left(\mathcal{U}_{n}: n \in \mathbb{N}\right)$ of collections of open sets in $X$ such that $\bar{A} \subset \cup \mathcal{U}_{n}, n \in \mathbb{N}$, there is a sequence $\left(\mathcal{V}_{n}: n \in \mathbb{N}\right)$ such that for each $n \in \mathbb{N}, \mathcal{V}_{n}$ is a finite subset of $\mathcal{U}_{n}$ and for each $x \in A, x \in \operatorname{St}\left(\cup \mathcal{V}_{n}, \mathcal{U}_{n}\right)$ for all but finitely many $n$.

(2) set strongly star Hurewicz property (shortly, set-SSH property) if for each nonempty subset $A$ of $X$ and each sequence $\left(\mathcal{U}_{n}: n \in \mathbb{N}\right)$ of collections of open sets in $X$ such that $\bar{A} \subset \cup \mathcal{U}_{n}, n \in \mathbb{N}$, there is a sequence $\left(F_{n}: n \in \mathbb{N}\right)$ of finite subsets of $\bar{A}$ such that for each $x \in A, x \in \operatorname{St}\left(F_{n}, \mathcal{U}_{n}\right)$ for all but finitely many $n$.

Definition 1.3 ([5,14]). A space $X$ is said to be:

(1) starcompact (shortly, SC) if for each open cover $\mathcal{U}$ of $X$, there is a finite subset $\mathcal{V}$ of $\mathcal{U}$ such that $X=\operatorname{St}(\cup \mathcal{V}, \mathcal{U})$.

(2) strongly starcompact (shortly, SSC) if for each open cover $\mathcal{U}$ of $X$, there is a finite subset $F$ of $X$ such that $X=\operatorname{St}(F, \mathcal{U})$.

In a similar way, Kočinac, Konca and Singh [13] considered the following spaces. 
Definition 1.4. A space $X$ is said to be:

(1) set starcompact (shortly, set-SC) if for each nonempty subset $A$ of $X$ and each open cover $\mathcal{U}$ of $\bar{A}$, there is a finite subset $\mathcal{V}$ of $\mathcal{U}$ such that $A=\operatorname{St}(\cup \mathcal{V}, \mathcal{U}) \cap A$.

(2) set strongly starcompact (shortly, set-SSC) if for each nonempty subset $A$ of $X$ and each open cover $\mathcal{U}$ of $\bar{A}$, there is a finite subset $F$ of $\bar{A}$ such that $A=\operatorname{St}(F, \mathcal{U}) \cap A$.

It is clear, by the definition, that every set strongly starcompact space is set starcompact.

Theorem 1.5 ([13]). Every countably compact space is set strongly starcompact.

Corollary 1.6 ([13]). Every countably compact space is set starcompact.

\section{Examples}

In this section, we give some examples showing the relationships between set star Hurewicz, set strongly star Hurewicz and other related spaces. Some of these examples can be found in the literature, and we establish their additional properties.

Lemma 2.1. Every Hurewicz space is set strongly star-Hurewicz.

Proof. Let $X$ be a Hurewicz space. Let $A$ be any nonempty subset of $X$ and $\left(\mathcal{U}_{n}: n \in \mathbb{N}\right)$ be a sequence of collections of open sets in $X$ such that $\bar{A} \subset \cup \mathcal{U}_{n}$. Since closed subset of Hurewicz space is Hurewicz, thus $\bar{A}$ is Hurewicz. Therefore there exists a sequence $\left(\mathcal{V}_{n}: n \in \mathbb{N}\right)$ such that for each $n \in \mathbb{N}, \mathcal{V}_{n}$ is a finite subset of $\mathcal{U}_{n}$ and for each $x \in \bar{A}$, $x \in \cup \mathcal{V}_{n}$ for all but finitely many $n$. Choose $x_{V} \in \bar{A} \cap V$ for each $V \in \mathcal{V}_{n}$. For each $n \in \mathbb{N}$, let $F_{n}=\left\{x_{V}: V \in \mathcal{V}_{n}\right\}$. Hence each $F_{n}$ is a finite subset of $\bar{A}$ and for each $x \in A$, $x \in \operatorname{St}\left(F_{n}, \mathcal{U}_{n}\right)$ for all but finitely many $n$.

From the definitions and the above lemma we have the following diagram.

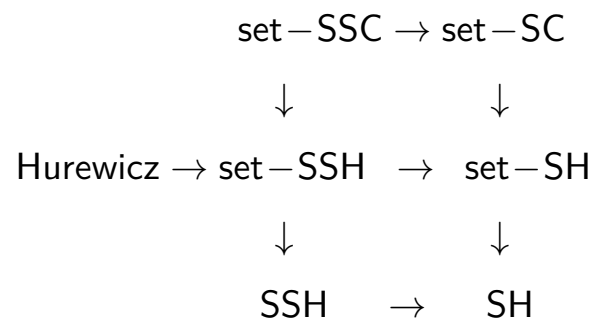

\section{Diagram 1}

However, the converse of the implications may not be true as we show by examples.

The following example shows that the implication Hurewicz $\Rightarrow$ set-SSH in Diagram 1 is not reversible.

Example 2.2. Every countably compact non-Lindelöf space is such an example. Such (Tychonoff) spaces are, for example, the ordinal space $\left[0, \omega_{1}\right)$, the long line [21, Example 45], the Novak space [21, Example 112].

The following example shows that the implications set-SSC $\Rightarrow$ set-SSH and set-SC $\Rightarrow$ set-SH in Diagram 1 are not reversible.

Example 2.3. There exists a Tychonoff set strongly star Hurewicz (hence, set star Hurewicz) space which is not set starcompact (hence, not set strongly starcompact). 
Indeed, let $X=D(\omega)$ be the countable discrete space. Since $X$ is $\sigma$-compact, it is Hurewicz and thus set strongly star Hurewicz. But $X$ is not set starcompact.

The following example shows that the implication set-SH $\Rightarrow \mathrm{SH}$ in Diagram 1 is not reversible (such an example was considered in several papers to obtain various counterexamples; see, for instance, $[4,19,20])$.

Example 2.4. There exists a Hausdorff star Hurewicz space which is not set star Hurewicz.

Proof. Let $X=Y \cup A \cup\{p\}$, where

$$
A=[0, \mathfrak{c}), \quad B=[0, \omega), Y=A \times B, p \notin Y .
$$

Topologize $X$ as follows:

(i) every point of $Y$ is isolated;

(ii) a basic neighborhood of $\alpha \in A$ is of the form

$$
U_{\alpha}(n)=\{\alpha\} \cup\{\langle\alpha, m\rangle: n<m\}
$$

(iii) a basic neighborhood of $p$ takes the form

$$
U(F)=\{p\} \cup \bigcup\{\langle\alpha, n\rangle: \alpha \in A \backslash F, n \in \omega\}
$$

for a countable subset $F$ of $A$. In [19, Example 2.7], Song proved that $X$ is star Hurewicz.

Now we show that $X$ is not set star Hurewicz. Consider $A=[0, \mathfrak{c})$, a closed discrete subset of $X$. For each $\alpha \in A$, let

$$
U_{\alpha}=\{\alpha\} \cup\{\langle\alpha, n\rangle: n \in B\} .
$$

Then $U_{\alpha}$ is open in $X$ by the construction of the topology of $\mathrm{X}$ and $U_{\alpha} \cap U_{\alpha^{\prime}}=\emptyset$ for $\alpha \neq \alpha^{\prime}$. For each $n \in \mathbb{N}$, let $\mathcal{U}_{n}=\left\{U_{\alpha}: \alpha \in A\right\}$. Then $\left(\mathcal{U}_{n}: n \in \mathbb{N}\right)$ is a sequence of open covers of $\bar{A}$. It is enough to show that there exists a point $\beta \in A$ such that $\beta \notin \operatorname{St}\left(\cup \mathcal{V}_{n}, \mathcal{U}_{n}\right)$ for all $n \in \mathbb{N}$, for any sequence $\left(\mathcal{V}_{n}: n \in \mathbb{N}\right)$ of finite subsets of $\mathcal{U}_{n}$. Let $\left(\mathcal{V}_{n}: n \in \mathbb{N}\right)$ be any sequence such that for each $n \in \mathbb{N}, \mathcal{V}_{n}$ is a finite subset of $\mathcal{U}_{n}$. For each $n \in \mathbb{N}$, $\mathcal{V}_{n}$ is finite, hence there exists $\alpha_{n}<\mathfrak{c}$ such that $U_{\alpha} \cap\left(\cup \mathcal{V}_{n}\right)=\emptyset$ for each $\alpha>\alpha_{n}$. Let $\alpha^{\prime}=\sup \left\{\alpha_{n}: n \in \mathbb{N}\right\}$. If we pick $\beta>\alpha^{\prime}$, then $U_{\beta} \cap\left(\cup \mathcal{V}_{n}\right)=\emptyset$ for each $n \in \mathbb{N}$. Since $U_{\beta}$ is the only element of $\mathcal{U}_{n}$ containing the point $\beta$ for each $n \in \mathbb{N}$, then $\beta \notin \operatorname{St}\left(\cup \mathcal{V}_{n}, \mathcal{U}_{n}\right)$ for all $n \in \mathbb{N}$. It shows that $X$ is not set star Hurewicz.

The following example shows that the implication set-SSH $\Rightarrow$ set-SH in Diagram 1 is not reversible.

Example 2.5. There exists a $T_{1}$ set star Hurewicz space which is not set strongly star Hurewicz.

Proof. Let $X=A \cup B$, where $A=\left\{a_{\alpha}: \alpha<\mathfrak{c}\right\}$ is any set with $|A|=\mathfrak{c}$ and $B$ is any set with $|B|=\omega$ such that any element of $B$ is not in $A$. Topologize $X$ as follows: for each $a_{\alpha} \in A$ and each finite subset $F \subset B,\left\{a_{\alpha}\right\} \cup(B \backslash F)$ is a basic open neighborhood of $a_{\alpha}$, and each element of $B$ is isolated.

First we show that $X$ is set star Hurewicz space. Let $C$ be any nonempty subset of $X$ and $\left(\mathcal{U}_{n}: n \in \omega\right)$ be any sequence of open covers of $\bar{C}$.

1. If $C \subset A$, then for each $n \in \omega$ and for each $a_{\alpha} \in C$, there exists $U_{\alpha, n} \in \mathcal{U}_{n}$ such that $a_{\alpha} \in U_{\alpha, n}$. Then for each $a_{\alpha} \in C$, we can find a finite set $F_{\alpha, n}$ such that $\left\{a_{\alpha}\right\} \cup\left(B \backslash F_{\alpha, n}\right) \subseteq U_{\alpha, n}$. Then it is clear that for each $n \in \omega$ and for each $\alpha \neq \alpha^{\prime}$, $U_{\alpha, n} \cap U_{\alpha^{\prime}, n} \neq \emptyset$. For each $n \in \omega$, let $\mathcal{V}_{n}=\left\{U_{\alpha, n}\right\}$. Then each $\mathcal{V}_{n}$ is a finite subset of $\mathcal{U}_{n}$ and hence for each $a_{\alpha} \in C, a_{\alpha} \in \operatorname{St}\left(\cup \mathcal{V}_{n}, \mathcal{U}_{n}\right)$ for all but finitely many $n$.

2. Let $C \subset B$. Since $B$ is countable, $B$ is set star Hurewicz. So, there are finite sets $\mathcal{V}_{n} \subset \mathcal{U}_{n}, n \in \mathbb{N}$, so that each $x \in C$ belongs to all but finitely many sets $\operatorname{St}\left(\cup \mathcal{V}_{n}, \mathcal{U}_{n}\right)$.

3. Finally, let $C \cap A=C_{1} \neq \emptyset$ and $C \cap B=C_{2} \neq \emptyset$. For each $n$ choose finite $\mathcal{V}_{n} \subset \mathcal{U}_{n}$ and $\mathcal{W}_{n} \subset \mathcal{U}_{n}$ such that each $x \in C_{1}$ belongs to all but finitely many sets $\operatorname{St}\left(\cup \mathcal{V}_{n}, \mathcal{U}_{n}\right)$ 
and each $x \in C_{2}$ belongs to all but finitely many sets $\operatorname{St}\left(\cup \mathcal{W}_{n}, \mathcal{U}_{n}\right)$. Evidently, then each $x \in C$ belongs to all but finitely many sets $\operatorname{St}\left(\cup\left(\mathcal{V}_{n} \cup \mathcal{W}_{n}\right), \mathcal{U}_{n}\right)$.

From the above three cases one concludes that $X$ is set star Hurewicz.

Similarly to the proof of Example 2.6, we can prove that $X$ is not set strongly star Hurewicz.

The following example shows that the implication set-SSH $\Rightarrow$ SSH in Diagram 1 consistently is not reversible.

Example 2.6. Assuming $\omega_{1}<\mathfrak{b}=\mathfrak{c}$, there exists a Tychonoff strongly star Hurewicz space $X$ which is not set strongly star Hurewicz.

Proof. Let $X=\psi(\mathcal{A})$ be the Isbell-Mrówka space with $|\mathcal{A}|=\omega_{1}$. Then $X$ is strongly star Hurewicz Tychonoff space (see Theorem 1.1).

Now we prove that $X$ is not set strongly star Hurewicz. Let $A=\mathcal{A}=\left\{a_{\alpha}: \alpha<\omega_{1}\right\}$. Then $A$ is closed subset of $X$. For each $\alpha<\omega_{1}$, let $U_{\alpha}=\left\{a_{\alpha}\right\} \cup\left(a_{\alpha}\right)$. For each $n \in \mathbb{N}$, let $\mathcal{U}_{n}=\left\{U_{\alpha}: \alpha<\omega_{1}\right\}$. Then $\left(\mathcal{U}_{n}: n \in \mathbb{N}\right)$ is a sequence of open covers of $\bar{A}$. It is enough to show that there exists a point $a_{\beta} \in A$ such that

$$
a_{\beta} \notin \operatorname{St}\left(F_{n}, \mathcal{U}_{n}\right) \text { for all } n \in \mathbb{N},
$$

for any sequence $\left(F_{n}: n \in \mathbb{N}\right)$ of finite subsets of $\bar{A}$. Let $\left(F_{n}: n \in \mathbb{N}\right)$ be any sequence of finite subsets of $\bar{A}$. Then there exists $\alpha^{\prime}<\omega_{1}$ such that $U_{\alpha} \cap\left(\bigcup_{n \in \mathbb{N}} F_{n}\right)=\emptyset$, for each $\alpha>\alpha^{\prime}$. Pick $\beta>\alpha^{\prime}$, then $U_{\beta} \cap F_{n}=\emptyset$ for each $n \in \mathbb{N}$. Since $U_{\beta}$ is the only element of $\mathcal{U}_{n}$ containing the point $a_{\beta}$ for each $n \in \mathbb{N}$, then $a_{\beta} \notin \operatorname{St}\left(F_{n}, \mathcal{U}_{n}\right)$ for all $n \in \mathbb{N}$. It shows that $X$ is not set strongly star Hurewicz.

Remark 2.7. (1) In [13, Example 5], Kočinac et al. gave an example of Tychonoff set starcompact space $X$ that is not set strongly starcompact. This shows that the implication set-SSC $\Rightarrow$ set-SC in Diagram 1 is not reversible.

(2) It is known that there are star Hurewicz spaces which are not strongly star Hurewicz (see [18]). This shows that the implication $\mathrm{SSH} \Rightarrow \mathrm{SH}$ in Diagram 1 is not reversible.

\section{Results}

In some classes of spaces certain properties from Diagram 1 coincide. In [3] the following theorem was proved.

Theorem 3.1 ([3, Proposition 4.1]). If $X$ is a paracompact Hausdorff space, then $X$ is star Hurewicz if and only if $X$ is Hurewicz.

From Theorem 3.1 and Diagram 1, we have the following.

Theorem 3.2. If $X$ is a paracompact Hausdorff space, then the following statements are equivalent:

(1) $X$ is Hurewicz;

(2) $X$ is set strongly star Hurewicz;

(3) $X$ is strongly star Hurewicz;

(4) $X$ is set star Hurewicz;

(5) $X$ is star Hurewicz.

A space $X$ is metacompact (resp., meta-Lindelöf) if each open cover of $X$ has a pointfinite (resp., point-countable) open refinement.

The following theorem can be obtained from the fact that every strongly star Hurewicz metacompact space is a Hurewicz space (which may be easily proved as Theorem 2.4 in [9]). However, we give here a direct proof of the theorem. 
Theorem 3.3. Every set strongly star Hurewicz hereditarily metacompact space $X$ is a (set) Hurewicz space.

Proof. Let $A$ be a subset of $X$ and $\left(\mathcal{U}_{n}: n \in \mathbb{N}\right)$ be a sequence of covers of $\bar{A}$ by open sets in $X$. For every $n \in \mathbb{N}$ the set $\cup \mathcal{U}_{n}$ is metacompact. Let $\mathcal{V}_{n}$ be a point-finite open refinement of $\mathcal{U}_{n}, n \in \mathbb{N}$. As $X$ is set strongly star Hurewicz, there is a sequence $\left(F_{n}: n \in \mathbb{N}\right)$ of finite subsets of $\bar{A}$ such that for each $x \in A, x \in \operatorname{St}\left(F_{n}, \mathcal{V}_{n}\right)$ for all but finitely many $n$. Elements of each $F_{n}$ belongs to finitely many members $V_{n, 1}, \ldots, V_{n, k(n)}$ of $\mathcal{V}_{n}$. Let $\mathcal{W}_{n}=\left\{V_{n, 1}, \ldots, V_{n, k(n)}\right\}$. Then $\operatorname{St}\left(F_{n}, \mathcal{V}_{n}\right)=\bigcup \mathcal{W}_{n}$, so that we have for each $x \in A, x \in \cup \mathcal{W}_{n}$ for all but finitely many $n$. For each $W \in \mathcal{W}_{n}$ take an element $U_{W}$ of $\mathcal{U}_{n}$ such that $W \subset U_{W}$. Then, for each $n, \mathcal{H}_{n}=\left\{U_{W}: W \in \mathcal{W}_{n}\right\}$ is a finite subset of $\mathcal{U}_{n}$ and for each $x \in A, x \in \cup \mathcal{H}_{n}$ for all but finitely many $n$. It is easy to prove that this fact actually gives that $X$ is a Hurewicz space.

Since every set strongly star Hurewicz space is strongly star Hurewicz, we have the following corollary from [17, Theorem 2.11] and [17, Corollary 2.12].

Corollary 3.4. If $X$ is a set strongly star Hurewicz space, then the following statements are equivalent:

(1) $X$ is a meta-Lindelöf space.

(2) $X$ is a para-Lindelöf space.

(3) $X$ is a Lindelöf space.

We now explore preservation of set star Hurewicz and set strongly star Hurewicz spaces under basic topological constructions.

Observe that set star Hurewicz and set strongly star Hurewicz are not hereditary properties. The space $X$ in Example 2.6, shows that a closed subset of a Tychonoff set star Hurewicz space $X$ need not be set star Hurewicz. Indeed, the set $\mathcal{A}$ is a discrete closed subset of the space $X$ in Example 2.6 of uncountable cardinality $\omega_{1}$, so that it cannot be set star Hurewicz.

We already noted that the ordinal space $X=\left[0, \omega_{1}\right)$ is set strongly star Hurewicz. However, the subspace $Y=\{\alpha+1: \alpha$ is a limit ordinal $\}$ of $X$ is not set strongly star Hurewicz.

However, we have the following result about preservation of set star Hurewicz and set strongly star Hurewicz spaces.

Theorem 3.5. A clopen subspace of a set star Hurewicz (resp., set strongly star Hurewicz) space is also set star Hurewicz (resp., set strongly star Hurewicz).

Proof. We prove only the set strongly star Hurewicz case because the proof for set star Hurewicz case is quite similar.

Let $X$ be a set strongly star Hurewicz space and $Y \subset X$ be a clopen subspace. Let $A$ be any subset of $Y$ and $\left(\mathcal{U}_{n}: n \in \mathbb{N}\right)$ be any sequence of collections of open sets in $\left(Y, \tau_{Y}\right)$ such that for each $n \in \mathbb{N}, \mathrm{Cl}_{Y}(A) \subset \cup \mathcal{U}_{n}$. Since $Y$ is open, then $\left(\mathcal{U}_{n}: n \in \mathbb{N}\right)$ is a sequence of collections of open sets in $X$, and since $Y$ is closed, $\mathrm{Cl}_{Y}(A)=\mathrm{Cl}_{X}(A)$. Applying the fact that $X$ is set strongly star Hurewicz, we find a sequence $\left(F_{n}: n \in \mathbb{N}\right)$ of finite subsets of $\mathrm{Cl}_{\mathrm{X}}(A)$ such that for each $x \in A, x \in \operatorname{St}\left(F_{n}, \mathcal{U}_{n}\right)$ for all but finitely many $n$. Because of $\mathrm{Cl}_{Y}(A)=\mathrm{Cl}_{X}(A)$, we have $F_{n} \subset Y, n \in \mathbb{N}$. Then the (same) sequence $\left(F_{n}: n \in \mathbb{N}\right)$ witnesses for $\left(\mathcal{U}_{n}: n \in \mathbb{N}\right)$ that $Y$ is set strongly star Hurewicz.

We now consider (non)preservation of the set star Hurewicz and set strongly star Hurewicz properties under some sorts of mappings.

Theorem 3.6. A continuous image of a set star Hurewicz space is set star Hurewicz. 
Proof. Let $X$ be a set star Hurewicz space and $f: X \rightarrow Y$ be a continuous mapping from $X$ onto $Y$. Let $B$ be any nonempty subset of $Y$ and $\left(\mathcal{V}_{n}: n \in \mathbb{N}\right)$ be a sequence of open covers of $\bar{B}$. Let $A=f^{\leftarrow}(B)$. Since $f$ is continuous, for each $n \in \mathbb{N}, \mathcal{U}_{n}:=\left\{f^{\leftarrow}(V): V \in\right.$ $\left.\mathcal{V}_{n}\right\}$ is the collection of open sets in $X$ with

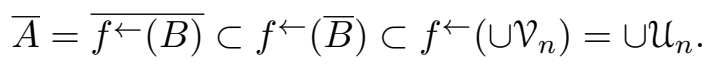

As $X$ is set star Hurewicz, there exists a sequence $\left(\mathcal{U}_{n}^{\prime}: n \in \mathbb{N}\right)$ such that for each $n \in \mathbb{N}$, $\mathcal{U}_{n}^{\prime}$ is a finite subset of $\mathcal{U}_{n}$ and for each $x \in A, x \in \operatorname{St}\left(\cup \mathcal{U}_{n}^{\prime}, \mathcal{U}_{n}\right)$ for all but finitely many $n$. Let $\mathcal{V}_{n}^{\prime}=\left\{V: f^{\leftarrow}(V) \in \mathcal{U}_{n}^{\prime}\right\}$. Then for each $n \in \mathbb{N}, \mathcal{V}_{n}^{\prime}$ is a finite subset of $\mathcal{V}_{n}$. Let $y \in B$. Then there exists $x \in A$ such that $f(x)=y$. Thus

$$
y=f(x) \in f\left(\operatorname{St}\left(\cup \mathcal{U}_{n}^{\prime}, \mathcal{U}_{n}\right)\right) \subset \operatorname{St}\left(\cup f\left(\left\{f^{\leftarrow}(V): V \in \mathcal{V}_{n}^{\prime}\right\}\right), \mathcal{V}_{n}\right)=\operatorname{St}\left(\cup \mathcal{V}_{n}^{\prime}, \mathcal{V}_{n}\right)
$$

for all but finitely many $n$. Thus $Y$ is a set star Hurewicz space.

We can prove the following theorem similarly to the proof of Theorem 3.6.

Theorem 3.7. A continuous image of a set strongly star Hurewicz space is set strongly star Hurewicz.

Now we give a result on preimages of set strongly star Hurewicz spaces. For this we need a new concept defined as follows. We call a space $X$ nearly set strongly star Hurewicz if for each $A \subset X$ and each sequence $\left(\mathcal{U}_{n}: n \in \mathbb{N}\right)$ of open covers of $X$ there is a sequence $\left(F_{n}: n \in \mathbb{N}\right)$ of finite subsets of $X$ such that for each $x \in A, x \in \operatorname{St}\left(F_{n}, \mathcal{U}_{n}\right)$ for all but finitely many $n$.

Theorem 3.8. Let $f: X \rightarrow Y$ be an open and closed, finite-to-one continuous mapping from a space $X$ onto a set strongly star Hurewicz space $Y$. Then $X$ is nearly set strongly star Hurewicz.

Proof. Let $A \subset X$ be any nonempty set and $\left(\mathcal{U}_{n}: n \in \mathbb{N}\right)$ be a sequence of open covers of $X$. Then $B=f(A)$ is a nonempty subset of $Y$. Let $y \in \bar{B}$. Then $f^{\leftarrow}(y)$ is a finite subset of $X$, and thus for each $n \in \mathbb{N}$, there is a finite subset $\mathcal{U}_{n}^{y}$ of $\mathcal{U}_{n}$ such that $f^{\leftarrow}(y) \subset \cup \mathcal{U}_{n}^{y}$ and $U \cap f^{\leftarrow}(y) \neq \emptyset$ for each $U \in \mathcal{U}_{n}^{y}$. Since $f$ is closed, there exists an open neighborhood $V_{n}^{y}$ of $y$ in $Y$ such that $f^{\leftarrow}\left(V_{n}^{y}\right) \subset \cup\left\{U: U \in \mathcal{U}_{n}^{y}\right\}$. Since $f$ is open, we can assume that

$$
V_{n}^{y} \subset \cap\left\{f(U): U \in \mathcal{U}_{n}^{y}\right\} .
$$

For each $n \in \mathbb{N}, \mathcal{V}_{n}=\left\{V_{n}^{y}: y \in \bar{B}\right\}$ is an open cover of $\bar{B}$. Since $Y$ is set strongly star Hurewicz, there exist a sequence $\left(F_{n}: n \in \mathbb{N}\right)$ of finite subsets of $\bar{B}$ such that for each $y \in B$,

$$
y \in \operatorname{St}\left(F_{n}, \mathcal{V}_{n}\right) \text { for all but finitely many } n .
$$

Since $f$ is finite-to-one, the sequence $\left(f^{\leftarrow}\left(F_{n}\right): n \in \mathbb{N}\right)$ is a sequence of finite subsets of $X$. Now we have to show that for each $x \in A$,

$$
x \in \operatorname{St}\left(f^{\leftarrow}\left(F_{n}\right), \mathcal{U}_{n}\right) \text { for all but finitely many } n .
$$

Let $x \in A$. Then there exist $n_{0} \in \mathbb{N}$ and $y \in B$ such that $y=f(x) \in V_{n}^{y}$ and $V_{n}^{y} \cap F_{n} \neq \emptyset$ for all $n \geq n_{0}$. Since

$$
x \in f^{\leftarrow}\left(V_{n}^{y}\right) \subset \bigcup\left\{U: U \in \mathcal{U}_{n}^{y}\right\},
$$

we can choose $U \in \mathcal{U}_{n}^{y}$ with $x \in U$. Then $V_{n}^{y} \subset f(U)$. Thus $U \cap f^{\leftarrow}\left(F_{n}\right) \neq \emptyset$ for all $n \geq n_{0}$. Hence

$$
x \in \operatorname{St}\left(f^{\leftarrow}\left(F_{n}\right), \mathcal{U}_{n}\right) \text { for all } n \geq n_{0} .
$$

Thus $X$ is nearly set strongly star Hurewicz. 
Following the above definition of nearly set strongly star Hurewicz spaces we call a space $\mathrm{X}$ nearly set star Hurewicz if for each nonempty $A \subset X$ and each sequence $\left(\mathcal{U}_{n}: n \in \mathbb{N}\right)$ of open covers of $X$ there is a sequence $\left(\mathcal{V}_{n}: n \in \mathbb{N}\right)$ such that for each $n \in \mathbb{N}, \mathcal{V}_{n}$ is a finite subset of $\mathcal{U}_{n}$ and for each $x \in A, x \in \operatorname{St}\left(\cup \mathcal{V}_{n}, \mathcal{U}_{n}\right)$ for all but finitely many $n$.

Similarly to the proof of Theorem 3.8, with necessary small modifications, we can prove the following.

Theorem 3.9. If $f: X \rightarrow Y$ is an open perfect mapping and $Y$ is a set star Hurewicz space, then $X$ is nearly set star Hurewicz.

From Theorem 3.9 we have the following corollary.

Corollary 3.10. If $X$ is a set star Hurewicz space and $Y$ is a compact space, then $X \times Y$ is nearly set star Hurewicz.

Remark 3.11. The product of two set star Hurewicz spaces need not be set star Hurewicz. In fact, there exist two countably compact spaces $X$ and $Y$ such that $X \times Y$ is not set star Hurewicz (even not set star Menger; see [13]). Moreover, there exist a countably compact (hence, set star Hurewicz) space $X$ and a Lindelöf space $Y$ such that $X \times Y$ is not set star Hurewicz (see [13]).

The following theorem is a version of Corollary 3.10. We say that a product $X \times Y$ is rectangular set star Hurewicz if for each set $A \times B \subset X \times Y$ and each sequence $\left(\mathcal{U}_{n}: n \in \mathbb{N}\right)$ of covers of $\overline{A \times B}$ by sets open in $X \times Y$ there are finite sets $\mathcal{V}_{n} \subset \mathcal{U}_{n}, n \in \mathbb{N}$, such that for each $z \in A \times B, z \in \operatorname{St}\left(\cup \mathcal{V}_{n}, \mathcal{U}_{n}\right)$ for all but finitely many $n$.

Theorem 3.12. If $X$ is a set star Hurewicz space and $Y$ is a compact space, then $X \times Y$ is rectangular set star Hurewicz.

Proof. Let $A=B \times C$ be any nonempty rectangular subset of $X \times Y$ and $\left(\mathcal{U}_{n}: n \in \mathbb{N}\right)$ be a sequence of collections of open sets in $X \times Y$ such that $\bar{A}=\bar{B} \times \bar{C} \subset \bigcup \mathcal{U}_{n}, n \in \mathbb{N}$. For each $x \in \bar{B},\{x\} \times \bar{C}$ is a compact subset of $X \times Y$. Therefore, for each $n \in \mathbb{N}$, there is a finite subset $\left\{U_{n, 1}^{x} \times V_{n, 1}^{x}, \ldots, U_{n, m(x)}^{x} \times V_{n, m(x)}^{x}\right\}$ of $\mathcal{U}_{n}$ such that $\{x\} \times \bar{C} \subset \bigcup_{i=1}^{m(x)}\left(U_{n, i}^{x} \times V_{n, i}^{x}\right)$. For each $n \in \mathbb{N}$, define $W_{n}^{x}=\bigcap_{i=1}^{m(x)} U_{n, i}^{x}$. Each $W_{n}^{x}$ is an open subset of $X$ containing $x$ and

$$
\{x\} \times \bar{C} \subset \bigcup\left\{W_{n}^{x} \times V_{n, i}^{x}: 1 \leq i \leq m(x)\right\} \subset \bigcup\left\{U_{n, i}^{x} \times V_{n, i}^{x}: 1 \leq i \leq m(x)\right\} .
$$

Then for each $n \in \mathbb{N}, \mathcal{W}_{n}=\left\{W_{n}^{x}: x \in \bar{B}\right\}$ is an open cover of $\bar{B}$. Since $X$ is set star Hurewicz, for each $n \in \mathbb{N}$, there is finite set $\mathcal{W}_{n}^{\prime}=\left\{W_{x_{j}}: 1 \leq j \leq r_{n}\right\}$ of $\mathcal{W}_{n}$ such that for each $b \in B, b \in \operatorname{St}\left(\cup \mathcal{W}_{n}^{\prime}, \mathcal{W}_{n}\right)$ for all but finitely many $n$. For each $n \in \mathbb{N}$, let

$$
\mathcal{U}_{n}^{\prime}=\left\{U_{n, i}^{x_{j}} \times V_{n, i}^{x_{j}}: 1 \leq i \leq n\left(x_{j}\right), 1 \leq j \leq r_{n}\right\} .
$$

Then $\mathcal{U}_{n}^{\prime}$ is a finite subset of $\mathcal{U}_{n}$. Hence for each $a \in A, a \in\left(\operatorname{St}\left(\cup \mathcal{W}_{n}^{\prime}, \mathcal{W}_{n}\right) \cap B\right) \times \bar{C} \subset$ $\operatorname{St}\left(\cup \mathcal{U}_{n}^{\prime}, \mathcal{U}_{n}\right)$ for all but finitely many $n$. Thus $X \times Y$ is rectangular set star Hurewicz.

Acknowledgment. The authors are deeply grateful to the referee for his/her careful reading of the paper and for a number of useful comments and remarks which improved the presentation of the paper.

\section{References}

[1] A.V. Arhangel'kii, A generic theorem in the theory of cardinal invariants of topological spaces, Comment. Math. Univ. Carol. 36, 303-325, 1995.

[2] M. Bonanzinga and M.V. Matveev, Some covering properties for $\psi$-spaces, Mat. Vesnik 61, 3-11, 2009. 
[3] M. Bonanzinga, F. Cammaroto and Lj.D.R. Kočinac, Star-Hurewicz and related properties, Appl. Gen. Topol. 5, 79-89, 2004.

[4] G. Di Maio and Lj.D.R. Kočinac, A note on quasi-Menger and similar spaces, Topology Appl. 179, 148-155, 2015.

[5] E.K. van Douwen, G.K. Reed, A.W. Roscoe and I.J. Tree, Star covering properties, Topology Appl. 39, 71-103, 1991.

[6] R. Engelking, General Topology, PWN, Warszawa, 1977.

[7] W. Hurewicz, Über die Verallgemeinerung des Borelshen Theorems, Math. Z. 24, 401-425, 1925.

[8] W. Hurewicz, Über Folgen stetiger Functionen, Fund. Math. 9, 193-204, 1927.

[9] Lj.D.R. Kočinac, Star-Menger and related spaces, Publ. Math. Debrecen 55, 421-431, 1999.

[10] Lj.D.R. Kočinac, Star-Menger and related spaces II, Filomat 13, 129-140, 1999.

[11] Lj.D.R. Kočinac, Addendum to: "Variations of classical selection principles: an overview", Quaest. Math., 2020. DOI: 10.2989/16073606.2020.1779501

[12] Lj,D.R. Kočinac and Ş. Konca, Set-Menger and related properties, Topology Appl. 275, Article No. 106996, 2020.

[13] Lj.D.R. Kočinac, Ş. Konca and S. Singh, Set star-Menger and set strongly star-Menger spaces, Math. Slovaca, in press.

[14] M.V. Matveev, A survey on star covering properties, Topology Atlas, Preprint No. $330,1998$.

[15] S. Mrówka, On completely regular spaces, Fund. Math. 41, 105-106, 1954.

[16] S. Singh, Remarks on set-Menger and related properties, Topology Appl. 280, Art. No. 107278, 2020.

[17] Y.K. Song, Remarks on strongly star-Hurewicz spaces, Filomat 27, 1127-1131, 2013.

[18] Y.K. Song, Remarks on star-Hurewicz spaces, Bull. Polish Acad. Sci. Math. 61, 247$255,2013$.

[19] Y.K. Song, On star-K-Hurewicz spaces, Filomat 31, 1129-1285, 2017.

[20] Y.K. Song and Y.Y. Zhang, Some remarks on almost Lindelöf spaces and weakly Lindelöf spaces, Mat. Vesnik 62, 77-83, 2010.

[21] L.A. Steen and J.A. Seebach, Counterexamples in Topology, Dover Publications Inc., 1996. 